CORRECTION

https://doi.org/10.1038/s41586-019-1490-y

\title{
Author Correction: Distinct modes of mitochondrial metabolism uncouple $T$ cell differentiation and function
}

Will Bailis, Justin A. Shyer, Jun Zhao, Juan Carlos Garcia Canaveras, Fatimah J. Al Khazal, Rihao Qu, Holly R. Steach, Piotr Bielecki, Omair Khan, Ruaidhri Jackson, Yuval Kluger, Louis J. Maher III, Joshua Rabinowitz, Joe Craft \& Richard A. Flavell

Correction to: Nature https://doi.org/10.1038/s41586-019-1311-3, published online 19 June 2019.

In this Letter, the 'Competing interests' statement should have stated: "R.A.F. is a founder, shareholder and advisor for Rheos Medicines, Inc". The original Letter has been corrected online. 